\title{
Determinant of malaria service readiness at health facility: Evidence from 2018 Service Availability and Readiness Assessment: Cross sectional study
}

Gebeyaw Molla ( $\sim$ gebeyawmolla48@gmail.com)

Ethiopian Public Health Institute https://orcid.org/0000-0002-1196-7458

Theodros Getachew

Ethiopian Public Health Institute

Atkure Defar

Ethiopian Public Health Institute

Tigist Shumet

Ethiopian Public Health Institute

Girum Taye

Ethiopian Public Health Institute

Misrak Getenet

Ethiopian Public Health Institute

Tefera Tadele

Ethiopian Public Health Institute

Habtamu Teklie

Ethiopian Public Health Institute

Geremew Gofa

Ethiopian Public Health Institute

Ambaye Tadese

Ethiopian Public Health Institute

Abebe Bekele

Ethiopian Public Health Institute

Research

Keywords: Malaria, Service availability, and readiness

Posted Date: November 28th, 2019

DOI: https://doi.org/10.21203/rs.2.17893/v1

License: (c) (1) This work is licensed under a Creative Commons Attribution 4.0 International License. Read Full License 


\section{Abstract}

Background: Globally, an estimated 3.3 billion people are at the risk of malaria. The majority of cases have occurred in the African Region. This study aims to assess the determinant of malaria service readiness at the health facility level.

Method: Data from the 2018 Service Availability and Readiness Assessment was used for this analysis. The study was a facility-based cross-sectional study and a stratified sampling technique was used. Data was collected from October - December 2017. Mean readiness score was used by computing six tracer items (Available of at least one trained staff for malaria diagnosis \& treatment, Available malaria diagnosis \& treatment guideline, Malaria diagnostic capacity, First-line anti-malarial drug in-stock, Paracetamol cap/tab, and ITN). Linear regression was used to identify factors associated with the mean readiness score of health facility to provide malaria service. The proportion test was used to check any change between 2016 and 2018 malaria service readiness.

Result: A total of 764 facilities were included in the study, of these only $682(89.3 \%)$ of facilities were provide malaria service. Eighty-nine percent of facilities offer diagnosis or treatment of malaria service and $70 \%$ of the facility diagnosis malaria by clinical symptom followed by microscopy (67\%) and Rapid Diagnostic Tests (RDT) (46\%). Only $3 \%$ facilities had all the six tracer items. Hospitals and health centers had the availability of tracer items above the average mean readiness score (52\%). Facilities managed by other than public authorities were had lower mean score readiness for malaria service compared with those managed by the public. Higher \& medium clinics, health posts, and Lower clinics were had lower mean score readiness for malaria service compared with hospitals. A significant change was not observed for malaria service readiness of tracer items between 2016 and 2018 ( $p$-value=0.732).

Conclusion: The study revealed that higher \& medium clinics, lower clinics, health posts, facilities managed by other authorities and region were the determinate factor of malaria service readiness.Keyword: Malaria, Service availability, and readiness

\section{Background}

Malaria is a life-threatening disease caused by the protozoan parasite of the genus Plasmodium which is transmitted by female Anopheles mosquitoes through biting (1). There are four human malaria parasite species which are Plasmodium falciparum, Plasmodium vivax, Plasmodium malaria and Plasmodium ovale (2). Plasmodium falciparum is the most dangerous and responsible for the majority of malaria-related deaths $(2,3)$. Malaria is a preventable and curable disease that remains an important cause of illness and death in children and adults (1).

Malaria has a significant effect on the health and wealth of individuals as well as nations (4). In pregnancy, it imposes a serious threat to the mother, fetus, and neonate (4) and one of the main reasons that children miss school and adults miss work and it hampers further educational achievement, contributes to food insecurity and entrenches poverty $(4,5)$.

According to the 2017 global malaria report indicates that more than 3.3 billion people were at risk of malaria (6). Of these, the majority of cases (92\%) were found in the African Region followed by South-East Asia Region (5\%) and the Eastern Mediterranean Region (2\%) (6). More than 480 million malaria cases were reported from sub-Saharan Africa (5). Malaria is one of the fatal causes of death that affect the nation of the country. In the region of Africa, $2017 \mathrm{global}$ malaria reports show that there are an estimated 435,000 deaths among these $61 \%$ were children (6).

Malaria is a major public health problem in Ethiopia. It is more dominate in the area of altitude below 2000 meters above sea level. In Ethiopia, more than twothirds of the population live an area that is highly affected by malaria (7). About $60 \%$ of the population living in this area is at risk for malaria and more than 1.5 million malaria cases are reported annually (8-10). Based on the President's Malaria Initiative annual performance report in $2015,2.2$ million cases and 662 deaths were reported (11). According to the Ethiopian Federal Ministry of Health Public Health Emergency Management (PHEM) report in 2018 shows that more than 1.2 million malaria cases and 158 deaths were reported annually. Out of these cases, $88 \%$ were confirmed cases through microscopy or rapid diagnostic tests (RDT), the majority (83\%) were laboratory-confirmed Plasmodium falciparum and 17\% were Plasmodium vivax cases (10).

As compared to the previous year, there is a significant reduction of a new case of malaria in Ethiopia. The number of new cases of malaria declined from 2.8 million in 1990 and 621,345 in 2015 and malaria death also reduced from 30,323 in 1990 to 1,561 in 2015. Age-standardized mortality rate declined by 96.5\% between 1990 and 2015 and the number of disability-adjusted life years lost (DALY) due to malaria decreased from 2.2 million in 1990 to 0.18 million in 2015 , with a total reduction of $91.7 \%$ (12). This was achieved by ensuring the availability of rapid diagnostic tests, anti-malaria drugs, trained health care workers, diagnostic capacity at the health facility level and high coverage of ITNs distribution and spraying of households (13). But, despite this reduction or improvement, malaria remains among the 10 most common causes of death and serious public health problem in Ethiopia (14). The possible barriers to achieving a further reduction in disease burden might be associated with service availability and readiness of each level of the health facility and community involvement in prevention activities.

Due to this global burden of malaria and the previous rapid signs of progress, WHO developed a Global technical strategy for malaria 2016-2030 with different milestones for measuring progress in 2020 and 2025 (2). Through this, all countries set their own national or sub-national targets to accelerate activities for eliminating malaria transmission and prevention and its re-establishment (15). The National Malaria Prevention, Control and Elimination Program (NMCP) strategy (NSP 2014-2020) in Ethiopia aim to achieve the goals of near-zero malaria deaths, reduction of malaria cases by $75 \%$ from a baseline of 2013 and elimination of malaria in the selected low transmission areas (11). To achieve these goal and the stated objectives, the NMCP will appropriately planned and targeted delivery of essential malaria interventions, including: early diagnose of suspected malaria, treatment of confirmed malaria cases with effective anti-malarial drug, and application of appropriate vector control interventions, particularly the use of insecticide-treated nets (ITN) \& indoor residual spraying (IRS) (6). Ethiopia develops a strategic plan to eliminate malaria by 2020 and to eradicate by 2030 , and go for a sub-national malaria elimination program. Therefore, there is a critical need for having well-trained health care workers, availability of adequate logistics and supply to offer 
malaria diagnosis and treatment service at all times in each level of health facility by strengthening the public-private partnership. To achieve those strategic goals, availability and readiness of health facilities for malaria diagnosis and treatment services are mandatory. Thus, the aim of this study was to assess the determinate of malaria service the availability and readiness of the health facility.

\section{Methods}

\section{Study design and area, sample size and sampling technique}

A facility-based cross-sectional study was conducted in nine regions (Tigray, Afar, Amhara, Oromia, Somali, Benishangul-Gumuz, SNNP, Gambella, and Harari) and two city administrations (Addis Ababa and Dire Dawa) of Ethiopia. Data was collected from October - December 2017. A stratified sampling technique was used to select the facility. Through this, all health facilities were stratified by region. From all-region and administrative cities, all hospitals and selected health centers, clinics and health posts were included in the sample. The total sample size for the study is 764 .

Data source: Data was obtained from Ethiopia Service Availability and Readiness Assessment 2018 and full of the methodology was hired over there.

Dependent variable: Readiness of health facility to provide malaria diagnosis and treatment service

Independent variable: region, location of health facility, managing authority, health facility type

\section{Operational definition:}

The readiness of health facilities is capacity of the health facility to provide malaria diagnosis and treatment. It measured by the availability of six tracer items: available of at least one trained staff for malaria diagnosis and treatment, available malaria diagnosis and treatment guideline, Malaria diagnostic capacity, First-line anti-malarial drug in-stock, Paracetamol cap/tab, and ITN.

\section{Data management and analysis}

English and Amharic translation of the facility inventory questionnaire was used to collect the data. The collected data was sent daily based on EPHI central server. Errors and inconsistencies were reviewed and checked at the central level correction were made accordingly. Internal consistency also validated through data cleaning.

Proportion, percentage and frequency distribution was used to present the result through tables. Mean readiness score is obtained from after computing the six tracer items (available of at least one trained staff for malaria diagnosis \& treatment, available malaria diagnosis \& treatment guideline, Malaria diagnostic capacity, First-line anti-malarial drug in-stock, Paracetamol cap/tab, and ITN) of malaria service. Multiple linear regression analyses were used to identify factors related to mean readiness score trace items for the provision of malaria diagnosis and treatment service. In linear regression analysis, all independent variables with a p-value of less than 0.25 in simple linear regression were included in multiple linear regression backward models. Finally, variables with P-value $<0.05$ were considered as significant predictors of the outcome variable. The Proportion test also used to compare the progress or any change of health facility readiness on the provision of malaria service between 2016 and 2018 Service Availability and Readiness Assessment using the overall mean score of tracer item.

\section{Results}

\section{Health facility distribution}

Seven hundred sixty-four facilities were had selected for malaria service availability and readiness. Of these, $40 \%$ were Hospital, $21 \%$ Health center, $17 \%$ health post, $12 \%$ higher \& medium clinic and $9 \%$ were a lower clinic. Concerning the regional distribution of health facilities, a large proportion of health facilities were found in the Oromia region 16\% (125), Amhara region 15\% (114) and SNNP region 14\% (105). Seventy-one percent of health facilities were public facilities and $66 \%$ were found in Urban (Table 1).

Table 1: Distribution of health facility across Region, managing authority and location, in Ethiopia $2018(\mathrm{~N}=764)$.

\section{Malaria Service Availability of health facility}

Nationally malaria service is given in all the tier health systems of each health facility. From the selected facilities for malaria service availability and readiness assessment, $89 \%$ of facilities offered malaria diagnosis or treatment service. Facilities found in the Gambela region offered malaria service and $46 \%$ of facilities found in this region were diagnosis malaria by microscopy through Rapid Diagnostic Test (RDT) (76\%) and clinical symptom (83\%). Almost all (98\%) hospitals and health centers provide malaria diagnosis or treatment service and 53\% of lower clinics offer malaria service. Except for RDT service (35\%), more than $75 \%$ of malaria service was available in an urban facility. Seventy-two percent of facilities managed by other than the public were diagnosing malaria through clinical symptoms (Table 2)..

Table 2: Malaria service availability by Region, Facility type, Managing authority and Urban/Rural in Ethiopia 2018(N = 764).

Page 3/10 


\section{The readiness of the health facility to provide Malaria diagnosis and treatment}

The readiness of health facility to offer malaria diagnosis or treatment service has assessed by the availability of the six tracer items (Table 3).. Six hundred eighty- two facilities were assessed for malaria readiness service based on the availability of tracer items. Of which, $85 \%$ of facilities were had malaria diagnostic capacity, $72 \%$ were had Paracetamol cap/tab and 65\% were had first anti-malaria drug. Only $19 \%$ of the facilities were had ITN and lower clinics were had no ITN service. Eighty-two percent and $58 \%$ of health posts had malaria diagnostic capacity through RDT and first-line anti-malarial drugs in stock respectively. Facilities managed by the public authority were had more likely availability of all tracer items compared with facilities managed by others (4\% Vs $1.7 \%$ ). Seven percent of the facility found in the Tigray region had availability of all tracer items. Nearly, $7 \%$ of the health centers and $4 \%$ of hospitals had the availability of all malaria tracer items but the rest health facilities were not had all tracer items. Overall, $3 \%$ (23) facilities were had all the six tracer items, $40 \%$ (274) were having more than three tracer items and nearly $4 \%$ (25) of facilities were had no malaria tracer items. The availability of tracer items was slightly higher than half, while $59 \%$ of Hospitals and nearly $62 \%$ of health centers were had the availability of tracer items above the average mean score readiness (52\%) (Table 3)..

Table 3: Distribution of malaria service tracer items by Region, Facility type, Managing authority and Location of the facility, Ethiopia 2018 ( $\mathrm{N}=682$.

\section{The Determinant of Malaria service readiness}

Malaria service readiness score is assessed by computing the mean score of the six-tracer item of malaria service. Based on this, the mean score of the tracer item has taken as the dependent variable. In simple linear regression those independent variables which are Facility type (Higher \& medium clinic, Lower clinic, and health post), Facility managed by other than public, Region (Afar, Amhara, Oromia Somali, Benishangul-Gumz, SNNP, Harari and Addis Ababa) and Facility found in rural area were significantly associated with the mean score of tracer item for malaria service readiness. After these, variables had entered into multiple linear regressions using the backward model to avoid the possibility of confounding effect. Finally, Facility type (Higher \& medium clinic, Lower clinic, and health post), facility managed by other than public and region (Amhara, Oromia, Somali, SNNP, Harari and Addis Ababa) was the determinant of the mean score of tracer item for malaria service readiness. Based on the finding, higher \& medium clinic $(B=-0.2[95 \% \mathrm{Cl}$ : $-0.26,-0.14])$, health post $(B=-$ $0.33[95 \% \mathrm{Cl}:-0.38,-0.28])$ and lower clinic $(\mathrm{B}=-0.48[95 \% \mathrm{Cl}:-0.54,-0.41])$ were lower mean score of tracer item for malaria service readiness compared with Hospitals. Facility managed by other than public authority was lower mean score of tracer items for malaria service readiness $(\mathrm{B}=-0.07[95 \% \mathrm{Cl}:-0.18$, -0.01]) compared with those managed by public authority counterpart. The region also one of the determinate factors of mean score readiness of tracer items to provide malaria service. From those region Addis Ababa $(B=-0.17[95 \% \mathrm{Cl}:-0.24,-0.11]), \operatorname{SNNP}(\mathrm{B}=-0.13[95 \% \mathrm{Cl}$ : $-0.19,-0.07])$, Oromia $(\mathrm{B}=-$ $0.12[95 \% \mathrm{Cl}:-0.18,-0.06])$, Somali $(\mathrm{B}=-0.12[95 \% \mathrm{Cl}:-0.19,-0.05])$, Harari $(\mathrm{B}=-0.1[95 \% \mathrm{Cl}:-0.18,-0.02])$ and $\mathrm{Amhara}$ region $(\mathrm{B}=-0.09[95 \% \mathrm{Cl}:(-0.15$, -0.03]) were had lower mean score of tracer item for malaria service readiness as compared with Tigray region (Table 4).

Table 4: Determinate of the mean score of tracer item for malaria service readiness, in Ethiopian health facilities, 2018.

\section{Discussion}

Health facility readiness to offer malaria diagnosis or treatment service is basic and boldly seen in each level of health facility to control, eliminate and eradicate malaria from its public health problem. At $5 \%$ level of significant, facility type (Higher \& medium clinic, Lower clinic, and health post), facility managed by other than public authority and region (Amhara, Oromia Somali, SNNP, Harari and Addis Ababa) were the determinant of mean score readiness of tracer item for malaria service. The current study revealed that $89 \%$ of health facilities offered malaria diagnosis or treatment services. Which is higher than another study conducted in Ethiopia (81\%) (16), in Somalia (57\%) (17) but lower than the study conducted in Tanzania (93\%) (18). This might be due to the presence of a security problem in Somalia to address malaria service and had a scale-up of different interventions in Ethiopia. Seventy percent of the facilities were conducted malaria diagnosis by clinical symptoms followed by microscopy (46\%) and RDT (67\%). Which is higher than from the previous study conducted in Ethiopia, $81 \%$ offer malaria diagnosis or treatment, $69 \%$ diagnosed by clinical symptom, $54 \%$ by microscopy and $39 \%$ by RDT(16) which is higher than study in Somalia, $32 \%$ by clinical symptom, $14 \%$ malaria by microscopy and $52 \%$ malaria by RDT(17). In this study, facilities that are managed by government authority were more likely to provide malaria service compared to non-governmental facilities (93\% Vs $81 \%$ ) which are in line with the study in Tanzania (95\% Vs $86 \%$ ) (18). But, the results from Somalia indicate that facilities managed by government authorities were less likely to offer malaria diagnosis or treatment service compared with facilities managed by others (59\% Vs $56 \%$ ) (17). Urban facilities in Ethiopia were more likely to offer malaria diagnosis or treatment service ( $92 \%$ Vs $82 \%)$ and also health centers were more likely to offer malaria service than health posts which is supported by other studies $(16,17)$.

Concerning malaria service readiness, $3 \%$ of the facilities have had the availability of all malaria tracer items which is nearly similar to the previous study in Ethiopia 3\% (16) and higher than the study conducted in Somalia 1\% (17). This difference might be used as unequal tracer items for malaria service readiness and instability of the country to improve malaria service through the implementation of malaria prevention and controlling strategy. Availability of trained health workers, anti-malaria drug and paracetamol tab in each level of health facility was mandatory. In the current study, $65 \%$ and $72 \%$ of the facilities have had the availability of ACT and Paracetamol tab in the facilities which is lower than different studies in Tanzania and Kenya (18-21). Nearly $30 \%$ of the facilities were had availability at least one trained health worker/Staff for malaria diagnosis and treatment service which is lower than the study connected in Tanzania 59\% (18) but higher than the previous study conducted in Ethiopia (17\%) and Nigeria $(24 \%)(16,22)$.

Overall, $52 \%$ of the facilities were ready to provide malaria service which is more likely to compared with study in Somalia (42\%) and less likely compare with Tanzania $(64 \%)(17,18)$. Facilities which are managed by other than public authority had lower mean score readiness of trace item by $7 \%$ compared with those facility managed by public authority. The significant regional difference was observed on the mean score readiness of the tracer item. This study 
revealed that facilities found in Addis Ababa, SNNP, Oromia, Somali and Amhara region were had lower the mean score readiness of trace item for malaria service by $17 \%, 13 \%, 12 \%, 12 \%, 10 \%$, and $9 \%$ compared with Tigray region respectively. In addition to this, there is a significant association between facility types on the mean score readiness of tracer items. Thus, the current study indicates that the mean score readiness of tracer items in the lower clinic, health post, and Higher \& Medium clinic were negatively associated with Hospitals. This might be the availability of well-trained health workers in hospitals and also hospitals were able to availed malaria service-related supply and logistic materials on their own. Overall there is no significant change in the readiness of health facility for malaria service between 2016 and 2018 Service Availability and Readiness Assessment (SARA) survey (P = 0.732 ). Therefore, the government and other stakeholders need more attention to the readiness of the health facility for malaria service provision to achieve the national malaria control strategy to elimination and eradication malaria by 2020 and 2030.

\section{Conclusions}

The mean score readiness of tracer items for malaria service is lower and only a few of the facilities were had the availability of all tracer items. The current study revealed that Facility type, managing authority, and region were the significant determinant of malaria service readiness. A significant change in malaria service readiness has not observed between the two 2016 and 2018 SARA surveys. Therefore, to achieve national malaria controlling and elimination strategy by 2020 and eradication by 2030 both the government and stakeholders should give more emphasis readiness of malaria service provision by availing all the trace items across the region and each level of the health facility. Managing authority should be critical to ensure facility readiness for malaria service. In addition, the public-private partnership will give more attention to improve malaria service at each level of the health facility.

\section{Abbreviations}

$B$ - Beta

Cl-Confident Interval

EPHI - Ethiopian Public Health Institute

$D A L Y$-Disability-Adjusted Life Years

ITN - Insecticide Treated Net

NMCP - National Malaria Control Program

RDT-Rapid Diagnostic Tests

SARA - Service Availability and Readiness Assessment

WHO - World Health Organization

\section{Declarations}

\section{Ethics approval and consent to participate}

Ethical Clearance was obtained from Ethiopian Public Health institute scientific ethical review office. Then officials at different levels in the study area were communicated through letters EPHI. Explanation of the necessary information about the purpose of the study, its procedure, assurance of confidentiality and privacy was informed. Written Informed Consent has taken from all facilities in charge and individual facility level identifier has not used. Data has maintained in a password protected EPHI server

\section{Consent to publication}

Not applicable

\section{Availability of data and materials}

The data of this study cannot be shared publicly due to the presence of sensitive (confidential) participants' information.

\section{Competing interests}

The authors declare that have no competing interests

\section{Funding}


Not applicable.

\section{Authors' Contributions}

GM has conceived, made a substantial contribution to the conception \& design of the study, clean the data, performed the data analysis, interpretation of the result, and drafting the manuscript. TW, AD, GT, and TS have been involved in data cleaning, coding, Analysis and revising the draft manuscript critically. TT and MG have been involved in data interpretation, assist manuscript preparation and critically review the draft of the manuscript content. $H T$, GG, AT and AB have been involved in collecting, collating the data, and assist the manuscript preparation. GM is the corresponding author submitting the manuscript for publication. All authors read and approved the final manuscript.

\section{Acknowledgments}

The authors are thankful to the Ethiopian Public Health Institute for their full cooperation in allowing this further analysis of the national data owned by the institute. It is our pleasure to acknowledge to Health system and reproductive health research directorate staff for their immense support by providing relevant comments during manuscript preparation. My pleasure also to express heart full thanks to Mr. Theodros Getachew for his willingness and invaluable support on the preparation of the paper

\section{References}

1. World Health Organizzation. Guidelines for the treatment of malaria-3rd edition [Internet]. Geneva; 2015. Available from: https://apps.who.int/iris/bitstream/handle/10665/162441/9789241549127_eng.pdf;jsessionid = 0CA55500AD77D56AE2C48A65A9515D95?sequence $=1$

2. World Health Organizzation. A framework for malaria elimination [Internet]. Geneva; 2017. Available from: https://apps.who.int/iris/bitstream/handle/10665/254761/9789241511988-eng.pdf?sequence = 1

3. World Health Organizzation. World malaria report 2017 [Internet]. Geneva; 2017. Available from: https://apps.who.int/iris/bitstream/handle/10665/259492/9789241565523-eng.pdf?sequence = 1

4. Karunamoorthi K. Global Malaria Burden: Socialomics Implications. J Soc. 2012;1(2):2-4.

5. US Agency international development. The President ' s Malaria Initiative Eleventh annual report to congress [Internet]. Washington; 2017. Available from: https://www.pmi.gov/docs/default-source/default-document-library/pmi-reports/2017-pmi-eleventh-annual-executive-summary.pdf

6. World Health Organizzation. World malaria report 2018 [Internet]. Geneva; 2018. Available from: https://creativecommons.org/ licenses/by-nc-sa/3.0/igo)

7. Communication for CHANGE. Essential Malaria Actions: Ethiopia [Internet]. 2012. Available from: https://www.cchangeprogram.org/sites/default/files/Ethiopia-EMA-Case-Study.pdf

8. Ministry of Health. Malaria Case Management Training Manual for Health Professionals in Ethiopia [Internet]. 2016. Available from: https://www.afro.who.int/sites/default/files/2017-06/9789241503976_eng.pdf

9. ICAP. Expands its Support for Ethiopia 's Ongoing Malaria Control Efforts [Internet]. Columbia; 2018. Available from: https://reliefweb.int/report/ethiopia/ica p-expands-its-support-ethiopia-s- ongoing-malaria-control-efforts\%0AREPORT

10. Ministry of Health. ANNUAL HEALTH SECTOR PERFORMANCE - EFY 2010(2017/18). [Internet]. Addis Ababa; 2018. Available from: https://www.itacaddis.org/docs/2017_11_10_09_48_31_ARM 2017.compressed.pdf

11. U.S Presedent's malaria initative. President's malaria initiative Ethiopia Malaria operational plan FY 2017 [Internet]. Available from: https://www.pmi.gov/docs/default-source/default-document-library/malaria-operational-plans/fy17/fy-2017-ethiopia-malaria-operational-plan.pdf? sfvrsn $=6$

12. Deribew A, Dejene T, Kebede B, Tessema GA, Melaku YA. Incidence, prevalence and mortality rates of malaria in Ethiopia from 1990 to 2015 : analysis of the global burden of diseases 2015. Malar J. 2017;16:1-7.

13. Federal Ministry of Health planning Department. Health and Health Related Indicators 2005 E. C ( 2012 / 2013). 2014; Available from: https://www.dktethiopia.org/sites/default/files/PublicationFiles/Health and Health Related Indicators 2005 E. C.pdf

14. Centers for Disease Control. Global Health - Ethiopia [Internet]. 2016. Available from: http://www.cdc.gov/globalhealth/countries/ethiopia/

15. World Health Organization. Regional malaria action plan 2016-2020 Towards a malaria- free Region [Internet]. Cairo; 2017. Available from: http://applications.emro.who.int/docs/EMROPUB_2017_EN_19546.pdf?ua = 1

16. Ministry of Health. Ethiopia Service Availability and Readiness Assessment [Internet]. Addis Ababa; 2016. Available from: https://www.washinhcf.org/documents/Final-SARA-Report-Jan-2017.pdf

17. Somali Health Authorities. Somali Service Availability and Readiness Assessment. [Internet]. 2016. Available from: https://www.humanitarianresponse.info/sites/www.humanitarianresponse.info/files/documents/files/somali_country_report_final_draft_30dec2016-.pdf

18. Ministry of Health. TANZANIA SERVICE AVAILABILITY AND READINESS ASSESSMENT ( SARA) [Internet]. 2013. Available from: https://ihi.eprints.org/2448/1/SARA_2012_Report.pdf

19. Njogu JN, International PS, Akhwale WS, Training I, Hamer DH. Health facility and health worker readiness to deliver new national treatment policy for malaria in Kenya. East Afr Med J. 2008;85(June):213-21. 
20. Ministry of Health. Kenya Service Availability and Readiness Assessment Mapping [Internet]. Nairobi Kenya; 2013. Available from: file:///C:/Users/user/Downloads/SARAM_KEN_report_2013.pdf

21. Group Act, Musuva A, Ejersa W, Kiptui R, Memusi D, Abwao E. The malaria testing and treatment landscape in Kenya: results from a nationally representative survey among the public and private sector. Malar J. 2017;16:1-13.

22. Ojo A, Aiyenigba B, Johnbull S, Onu A, Ipadeola O, Salihu M, et al. Malaria Diagnostic Service Availability - Mapping of Private Sector Service Delivery Outlets in 7 PMI / MAPS Supported States, Nigeria. 2014.

\section{Tables}

Table 1: Distribution of health facility across Region, managing authority and location, in Ethiopia 2018 (N=764).

\begin{tabular}{|c|c|c|c|c|c|c|c|c|c|c|c|}
\hline \multirow[t]{2}{*}{ riable } & \multicolumn{2}{|c|}{ Hospital } & \multicolumn{2}{|c|}{ Health Centre } & \multicolumn{2}{|c|}{ Health post } & \multicolumn{2}{|c|}{ Higher \& medium clinic } & \multicolumn{2}{|c|}{ Lower clinic } & \multirow[t]{2}{*}{ Total } \\
\hline & $\mathbf{n}$ & $\%$ & $\mathbf{n}$ & $\%$ & $\mathbf{n}$ & $\%$ & $\mathbf{n}$ & $\%$ & $\mathbf{n}$ & $\%$ & \\
\hline \multicolumn{12}{|l|}{ :gion } \\
\hline gray & 39 & 51 & 14 & 18 & 11 & 14 & 9 & 12 & 3 & 4 & 76 \\
\hline $\operatorname{ar}$ & 6 & 12 & 16 & 33 & 12 & 24 & 12 & 24 & 3 & 6 & 49 \\
\hline ahara & 65 & 57 & 17 & 15 & 16 & 14 & 4 & 4 & 12 & 11 & 114 \\
\hline omia & 75 & 60 & 16 & 13 & 16 & 13 & 1 & 1 & 17 & 14 & 125 \\
\hline mali & 11 & 19 & 16 & 28 & 13 & 23 & 17 & 30 & 0 & 0 & 57 \\
\hline :nishangul-Gumuz & 2 & 4 & 15 & 33 & 14 & 31 & 3 & 7 & 11 & 24 & 45 \\
\hline JNP & 58 & 55 & 16 & 15 & 16 & 15 & 6 & 6 & 9 & 9 & 105 \\
\hline imbella & 3 & 7 & 13 & 31 & 12 & 29 & 5 & 12 & 9 & 21 & 42 \\
\hline irrari & 5 & 14 & 8 & 23 & 11 & 31 & 10 & 29 & 1 & 3 & 35 \\
\hline dis Ababa & 32 & 42 & 22 & 29 & 0 & 0 & 17 & 22 & 6 & 8 & 77 \\
\hline riDawa & 7 & 18 & 11 & 28 & 11 & 28 & 9 & 23 & 1 & 3 & 39 \\
\hline \multicolumn{12}{|l|}{ anaging Authority } \\
\hline blic & 243 & 45 & 160 & 30 & 132 & 24 & 5 & 1 & 2 & 0 & 542 \\
\hline her & 60 & 27 & 4 & 2 & 0 & 0 & 88 & 40 & 70 & 32 & 222 \\
\hline \multicolumn{12}{|l|}{ ban/Rural } \\
\hline ban & 273 & 54 & 86 & 17 & 10 & 2 & 89 & 18 & 46 & 9 & 504 \\
\hline iral & 30 & 12 & 78 & 30 & 122 & 47 & 4 & 2 & 26 & 10 & 260 \\
\hline tal & 303 & 40 & 164 & 21 & 132 & 17 & 93 & 12 & 72 & 9 & 764 \\
\hline
\end{tabular}

Table 2: Malaria service availability by Region, Facility type, Managing authority and Urban/Rural in Ethiopia 2018 (N =764). 


\begin{tabular}{|c|c|c|c|c|c|c|c|c|c|c|c|c|c|c|c|}
\hline \multirow[t]{2}{*}{$\overline{\mathrm{e}}$} & \multicolumn{2}{|c|}{$\begin{array}{l}\text { Offer diagnosis or } \\
\text { treatment of malaria }\end{array}$} & \multicolumn{2}{|c|}{$\begin{array}{c}\text { Malaria } \\
\text { diagnosis }\end{array}$} & \multicolumn{2}{|c|}{$\begin{array}{c}\text { Malaria } \\
\text { diagnosis } \\
\text { testing }\end{array}$} & \multicolumn{2}{|c|}{$\begin{array}{l}\text { Malaria diagnosis by } \\
\text { clinical symptoms }\end{array}$} & \multicolumn{2}{|c|}{$\begin{array}{c}\text { Malaria } \\
\text { diagnosis by } \\
\text { RDT }\end{array}$} & \multicolumn{2}{|c|}{$\begin{array}{c}\text { Malaria diagnosis by } \\
\text { microscopy }\end{array}$} & \multicolumn{2}{|c|}{$\begin{array}{c}\text { Malaria } \\
\text { treatment }\end{array}$} & \multirow{2}{*}{$\begin{array}{c}\text { Total } \\
\mathrm{n}\end{array}$} \\
\hline & $\mathrm{n}$ & $\%$ & $\mathrm{n}$ & $\%$ & $\mathrm{n}$ & $\%$ & $\mathrm{n}$ & $\%$ & $\mathrm{n}$ & $\%$ & $\mathrm{n}$ & $\%$ & $\mathrm{n}$ & $\%$ & \\
\hline & 72 & 95 & 72 & 95 & 71 & 93 & 40 & 53 & 43 & 57 & 56 & 74 & 71 & 93 & 76 \\
\hline & 48 & 98 & 48 & 98 & 48 & 98 & 47 & 96 & 33 & 67 & 32 & 65 & 48 & 98 & 49 \\
\hline & 101 & 89 & 101 & 89 & 98 & 86 & 52 & 46 & 35 & 31 & 79 & 69 & 100 & 88 & 114 \\
\hline & 106 & 85 & 105 & 84 & 98 & 78 & 92 & 74 & 47 & 38 & 84 & 67 & 104 & 83 & 125 \\
\hline & 47 & 82 & 47 & 82 & 45 & 79 & 47 & 82 & 44 & 77 & 35 & 61 & 44 & 77 & 57 \\
\hline \multirow[t]{2}{*}{ ngul- } & 39 & 87 & 39 & 87 & 37 & 82 & 13 & 29 & 29 & 64 & 19 & 42 & 39 & 87 & 45 \\
\hline & 91 & 87 & 91 & 87 & 88 & 84 & 82 & 78 & 34 & 32 & 75 & 71 & 91 & 87 & 105 \\
\hline \multirow[t]{2}{*}{ la } & 42 & 100 & 40 & 95 & 38 & 90 & 35 & 83 & 32 & 76 & 20 & 48 & 42 & 100 & 42 \\
\hline & 32 & 91 & 32 & 91 & 31 & 89 & 31 & 89 & 13 & 37 & 22 & 63 & 31 & 89 & 35 \\
\hline jaba & 68 & 88 & 68 & 88 & 65 & 84 & 59 & 77 & 26 & 34 & 64 & 83 & 68 & 88 & 77 \\
\hline wa & 36 & 92 & 36 & 92 & 36 & 92 & 36 & 92 & 19 & 49 & 26 & 67 & 35 & 90 & 39 \\
\hline \multicolumn{16}{|l|}{ Type } \\
\hline l & 297 & 98 & 297 & 98 & 297 & 98 & 237 & 78 & 102 & 34 & 293 & 97 & 296 & 98 & 303 \\
\hline Jentre & 160 & 98 & 160 & 98 & 158 & 96 & 118 & 72 & 103 & 63 & 131 & 80 & 160 & 98 & 164 \\
\hline jost & 102 & 77 & 101 & 77 & 99 & 75 & 67 & 51 & 99 & 75 & 1 & 1 & 99 & 75 & 132 \\
\hline $\begin{array}{l}\mathrm{E} \\
\text { clinic }\end{array}$ & 85 & 91 & 85 & 91 & 85 & 91 & 78 & 84 & 37 & 40 & 84 & 90 & 82 & 88 & 93 \\
\hline linic & 38 & 53 & 36 & 50 & 16 & 22 & 34 & 47 & 14 & 19 & 3 & 4 & 36 & 50 & 72 \\
\hline \multicolumn{16}{|c|}{ ng Authority } \\
\hline & 502 & 93 & 501 & 92 & 496 & 92 & 375 & 69 & 273 & 50 & 369 & 68 & 499 & 92 & 542 \\
\hline & 180 & 81 & 178 & 80 & 159 & 72 & 159 & 72 & 82 & 37 & 143 & 64 & 174 & 78 & 222 \\
\hline \multicolumn{16}{|l|}{$\mathrm{n}$} \\
\hline & 463 & 92 & 463 & 92 & 448 & 89 & 377 & 75 & 178 & 35 & 427 & 85 & 456 & 90 & 504 \\
\hline & 219 & 84 & 216 & 83 & 207 & 80 & 157 & 60 & 177 & 68 & 85 & 33 & 217 & 83 & 260 \\
\hline & 682 & 89 & 679 & 89 & 655 & 86 & 534 & 70 & 355 & 46 & 512 & 67 & 673 & 88 & 764 \\
\hline
\end{tabular}

Table 3: Distribution of malaria service tracer items by Region, Facility type, Managing authority and Location of facility, Ethiopia 2018 (N=682) 


\begin{tabular}{|c|c|c|c|c|c|c|c|c|c|c|}
\hline $\begin{array}{l}\text { Guidelines } \\
\text { available } \\
\text { diagnosis } \\
\text { and } \\
\text { treatment } \\
\text { of malaria }\end{array}$ & $\begin{array}{l}\text { At least } 1 \\
\text { trained } \\
\text { staff } \\
\text { diagnosis } \\
\text { and } \\
\text { treatment } \\
\text { of malaria }\end{array}$ & $\begin{array}{c}\text { Malaria } \\
\text { diagnostic } \\
\text { capacity }\end{array}$ & $\begin{array}{l}\text { First-line } \\
\text { anti- } \\
\text { malarial } \\
\text { in-stock }\end{array}$ & $\begin{array}{c}\text { Paracetamol } \\
\text { cap/tab }\end{array}$ & ITN & $\begin{array}{c}\text { had no } \\
\text { tracer item }\end{array}$ & $\begin{array}{c}\text { had }>3 \text { tracer } \\
\text { items }\end{array}$ & $\begin{array}{c}\text { had all tracer } \\
\text { items }\end{array}$ & $\begin{array}{c}\text { Mean } \\
\text { availability } \\
\text { of tracer } \\
\text { items }\end{array}$ & Total \\
\hline$\%$ & $\%$ & $\%$ & $\%$ & $\%$ & $\%$ & $\%$ & $\%$ & $\%$ & & \\
\hline
\end{tabular}

\begin{tabular}{|c|c|c|c|c|c|c|c|c|c|c|c|c|c|c|c|c|c|c|c|c|}
\hline & $\mathbf{n}$ & $\%$ & $\mathrm{n}$ & $\%$ & $\mathbf{n}$ & $\%$ & $\mathrm{n}$ & $\%$ & $\mathrm{n}$ & $\%$ & $\mathrm{n}$ & $\%$ & $\mathbf{n}$ & $\%$ & $\mathbf{n}$ & $\%$ & $\mathbf{n}$ & $\%$ & & \\
\hline & 49 & 68.1 & 38 & 52.8 & 64 & 88.9 & 55 & 76.4 & 56 & 77.8 & 10 & 13.9 & 3 & 4.2 & 47 & 65.3 & 5 & 6.9 & $63.0 \%$ & 72 \\
\hline & 20 & 41.7 & 11 & 22.9 & 44 & 91.7 & 32 & 66.7 & 30 & 62.5 & 8 & 16.7 & 1 & 2.1 & 17 & 35.4 & 1 & 2.1 & $50.3 \%$ & 48 \\
\hline & 42 & 41.6 & 31 & 30.7 & 82 & 81.2 & 84 & 83.2 & 85 & 84.2 & 12 & 11.9 & 0 & 0 & 43 & 42.6 & 3 & 3 & 55.4 & 101 \\
\hline & 40 & 37.7 & 31 & 29.2 & 84 & 79.2 & 75 & 70.8 & 86 & 81.1 & 18 & 17.0 & 7 & 6.6 & 47 & 44.3 & 3 & 2.8 & $52.5 \%$ & 106 \\
\hline & 6 & 12.8 & 11 & 23.4 & 43 & 91.5 & 24 & 51.1 & 34 & 72.3 & 21 & 44.7 & 0 & 0 & 14 & 29.8 & 3 & 6.4 & $49.3 \%$ & 47 \\
\hline \multirow[t]{4}{*}{ jul-Gumz } & 7 & 17.9 & 6 & 15.4 & 31 & 79.5 & 29 & 74.4 & 21 & 53.8 & 15 & 38.5 & 3 & 7.7 & 13 & 33.3 & 1 & 2.6 & $46.6 \%$ & 39 \\
\hline & 36 & 39.6 & 17 & 18.7 & 80 & 87.9 & 64 & 70.3 & 72 & 79.1 & 14 & 15.4 & 3 & 3.3 & 31 & 34.1 & 2 & 2.2 & $51.8 \%$ & 91 \\
\hline & 17 & 40.5 & 23 & 54.8 & 34 & 81.0 & 27 & 64.3 & 20 & 47.6 & 12 & 28.6 & 4 & 9.5 & 21 & 50. & 3 & 7.1 & $52.8 \%$ & 42 \\
\hline & 17 & 53.1 & 9 & 28.1 & 29 & 90.6 & 14 & 43.8 & 13 & 40.6 & 1 & 3.1 & 0 & 0 & 8 & 25 & 0 & 0 & $43.2 \%$ & 32 \\
\hline ba & 35 & 51.5 & 7 & 10.3 & 61 & 89.7 & 18 & 26.5 & 51 & 75.0 & 0 & 0 & 2 & 2.9 & 14 & 20.6 & 0 & 0 & $42.2 \%$ & 68 \\
\hline z & 11 & 30.6 & 19 & 52.8 & 27 & 75.0 & 23 & 63.9 & 25 & 69.4 & 19 & 52.8 & 2 & 5.6 & 19 & 52.8 & 2 & 5.6 & $57.4 \%$ & 36 \\
\hline \multicolumn{21}{|l|}{ ype } \\
\hline & 130 & 43.8 & 85 & 28.6 & 269 & 90.6 & 240 & 80.8 & 289 & 97.3 & 42 & 14.1 & 1 & .3 & 144 & 48.5 & 12 & 4 & $59.2 \%$ & 297 \\
\hline ntre & 73 & 45.6 & 52 & 32.5 & 141 & 88.1 & 124 & 77.5 & 154 & 96.3 & 49 & 30.6 & 0 & 0 & 92 & 57.5 & 11 & 6.9 & $61.8 \%$ & 160 \\
\hline st & 30 & 29.4 & 45 & 44.1 & 84 & 82.4 & 59 & 57.8 & 0 & 0.0 & 38 & 37.3 & 5 & 4.9 & 25 & 24.5 & 0 & 0 & $41.8 \%$ & 102 \\
\hline medium & 44 & 51.8 & 19 & 22.4 & 72 & 84.7 & 19 & 22.4 & 37 & 43.5 & 1 & 1.2 & 4 & 4.7 & 13 & 15.3 & 0 & 0 & $37.6 \%$ & 85 \\
\hline \multirow[t]{6}{*}{ aic } & 3 & 7.9 & 2 & 5.3 & 13 & 34.2 & 3 & 7.9 & 13 & 34.2 & 0 & 0 & 15 & 39.5 & 0 & 0 & 0 & 0 & $14.9 \%$ & 38 \\
\hline & 206 & 41.0 & 169 & 33.7 & 441 & 87.8 & 397 & 79.1 & 387 & 77.1 & 124 & 24.7 & 6 & 1.2 & 241 & 48 & 20 & 4 & $57.2 \%$ & 502 \\
\hline & 74 & 41.1 & 34 & 18.9 & 138 & 76.7 & 48 & 26.7 & 106 & 58.9 & 6 & 3.3 & 19 & 10.6 & 33 & 18.3 & 3 & 1.7 & $37.6 \%$ & 180 \\
\hline & 191 & 41.3 & 121 & 26.1 & 398 & 86.0 & 305 & 65.9 & 384 & 82.9 & 59 & 12.7 & 13 & 2.8 & 187 & 40.4 & 15 & 3.2 & $52.5 \%$ & 463 \\
\hline & 89 & 40.6 & 82 & 37.4 & 181 & 82.6 & 140 & 63.9 & 109 & 49.8 & 71 & 32.4 & 12 & 5.5 & 87 & 39.7 & 8 & 3.7 & $51.1 \%$ & 219 \\
\hline & 280 & 41.1 & 203 & 29.8 & 579 & 84.9 & 445 & 65.2 & 493 & 72.3 & 130 & 19.1 & 25 & 3.7 & 274 & 40.2 & 23 & 3.4 & $52.1 \%$ & 682 \\
\hline
\end{tabular}

Table 4: Determinate of mean score of tracer item for malaria service readiness, in Ethiopian health facilities, 2018.

\begin{tabular}{|c|c|c|c|c|c|c|}
\hline \multirow[t]{2}{*}{ Variable } & \multirow[t]{2}{*}{ Mean score } & \multirow[t]{2}{*}{$\mathrm{SE}$} & \multicolumn{2}{|c|}{ Unadjusted coefficient } & \multicolumn{2}{|c|}{ Adjusted coefficient } \\
\hline & & & Beta & $95 \%$ CI & Beta & $95 \% \mathrm{CI}$ \\
\hline \multicolumn{7}{|l|}{ Facility type } \\
\hline Hospital (Ref) & 0.588 & 0.01 & & & & \\
\hline Health center & 0.607 & 0.02 & 0.02 & $(-0.02,0.06)$ & -0.03 & $(-0.07,0.02)$ \\
\hline Health post & 0.328 & 0.02 & -0.26 & $(-0.30,-0.22) * *$ & -0.33 & $(-0.38,-0.28) * *$ \\
\hline Higher \&medium clinic & 0.355 & 0.02 & -0.23 & $(-0.28,-0.19) * *$ & -0.2 & $(-0.26,-0.14) * *$ \\
\hline Lower clinic & 0.093 & 0.03 & -0.5 & $(-0.55,-0.44) * *$ & -0.48 & $(-0.54,-0.41) * *$ \\
\hline \multicolumn{7}{|l|}{ Managing authority } \\
\hline Public (Ref) & 0.547 & 0.01 & & & & \\
\hline Others & 0.315 & 0.02 & -0.22 & $(-0.26,-0.18) * *$ & -0.07 & $(-0.18,-0.01) *$ \\
\hline \multicolumn{7}{|l|}{ Region } \\
\hline Tigray (Ref) & 0.596 & 0.03 & & & & \\
\hline Afar & 0.493 & 0.05 & -0.103 & $(-0.19,-0.01) *$ & -0.03 & $(-0.10,0.04)$ \\
\hline Amhara & 0.493 & 0.04 & -0.104 & $(-0.18,-0.03) *$ & -0.09 & $(-0.15,-0.03) *$ \\
\hline Oromia & 0.456 & 0.04 & -0.14 & $(-0.21,-0.07) * *$ & -0.12 & $(-0.18,-0.06) * *$ \\
\hline Somali & 0.421 & 0.46 & -0.18 & $(-0.26,-0.09) * *$ & -0.12 & $(-0.19,-0.05) *$ \\
\hline Benishandul-Gumz & 0.415 & 0.05 & -0.18 & $(-0.28,-0.09) * *$ & -0.03 & $(-0.11,0.04)$ \\
\hline SNNP & 0.456 & 0.04 & -0.14 & $(-0.22,-0.06) * *$ & 0.13 & $(-0.19,-0.07) * *$ \\
\hline Gambella & 0.528 & 0.05 & -0.07 & $(-0.17, .03)$ & 0.07 & $(-0.01,0.14)$ \\
\hline Harrari & 0.4 & 0.05 & -0.196 & $(-0.3,-0.09) * *$ & -0.1 & $(-0.18,-0.02) *$ \\
\hline Addis Ababa & 0.394 & 0.04 & -0.2 & $(-0.29,-0.12) * *$ & -0.17 & $(-0.24,-0.11) * *$ \\
\hline DeriDawa & 0.543 & 0.05 & -0.05 & $(-0.15,0.05)$ & 0.02 & $(-0.06,0.09)$ \\
\hline \multicolumn{7}{|l|}{ Urban/Rural } \\
\hline Urban (Ref) & 0.489 & 0.01 & & & & \\
\hline Rural & 0.439 & 0.02 & -0.05 & $(-0.09,-0.01)^{*}$ & 0.02 & $(-0.02,0.06)$ \\
\hline
\end{tabular}

CI- Confidence Interval, SE-Standard Error, *P-value $<0.05, * *$ p-value $<0.001$, Ref-Reference 
Page 10/10 